International Journal of Engineering \& Technology, $7(4.37)(2018) 202-206$
International Journal of Engineering \& Technology
SPC
Website: www.sciencepubco.com/Index.Php/IJET
Research Paper

\title{
Fusion of Airborne Hyperspectral and WorldView2 Multispectral Images for Detailed Urban Land Cover Classification A case Study of Kuala Lumpur, Malaysia
}

\author{
Abbas Mohammed Noori ${ }^{1 *}$, Sumaya Falih Hasan², Qayssar Mahmood Ajaj ${ }^{3}$, Mustafa Ridha Mezaal ${ }^{4}$, Helmi Z. M. \\ Shafri ${ }^{5}$, Muntadher Aidi Shareef ${ }^{6}$ \\ ${ }_{1,2,3,6}$ Department of Surveying Techniques Engineering, Technical College of Kirkuk, Northern Technical University, Baghdad Road, \\ Kirkuk 36001, Iraq \\ ${ }^{4,5}$ Department of Civil Engineering, Faculty of Engineering, University Putra Malaysia, 43400 UPM, Serdang, Selangor, Malaysia \\ *Corresponding author E-mail: abbasrsgis@gmail.com
}

\begin{abstract}
Detecting the features of urban areas in detail requires very high spatial and spectral resolution in images. Hyperspectral sensors usually offer high spectral resolution images with a low spatial resolution. By contrast, multispectral sensors produce high spatial resolution images with a poor spectral resolution. Therefore, numerous fusion algorithms and techniques have been proposed in recent years to obtain high-quality images with improved spatial and spectral resolutions by sensibly combining the data acquired for the same scene. This work aims to exploit the extracted information from images in an effective way. To achieve this objective, a new algorithm based on transformation was developed. This algorithm primarily depends on the Gram-Schmidt process for fusing images, removing distortions, and improving the appearance of images. Images are first fused by using the Gram-Schmidt pansharpening method. The obtained fused image is utilized in the classification process in different areas by using support vector machine (SVM). The classification result is evaluated using a matrix of errors. The overall accuracy produced from the hyperspectral, multispectral and fused images was $72.33 \%$, $82.83 \%$, and $89.34 \%$, respectively. Results showed that the developed algorithm improved the image enhancement and image fusion. Moreover, the developed algorithm has the ability to produce an imaging product with high spatial resolution and high-quality spectral data.
\end{abstract}

Keywords: Fusion, Hyperspectral, Multispectral, Remote sensing, SVM, Gram-Schmidt, Pan Sharpening.

\section{Introduction}

Urban environments are composed of a broad range of manmade and natural surface materials that contribute to health, pollution, and microclimate in urban settings (Hamedianfar et al., 2014a). To ensure urban sustainability, comprehensive knowledge of the coverage and types of urban features is important essential (Hamedianfar et al., 2014b). Potential applications related to urban planning and management include mapping impervious surfaces for flood management, urban water quality and mapping of roof types for energy use and fire hazards (Woycheese et al., 1998). Remote sensing technology is a potential tool that can be used for dynamic monitoring of urban changes (Ajaj et al., 2017). Generally, a tradeoff exists between spatial and spectral resolutions due to the design of sensors. Hyperspectral (HS) images often have a high spectral resolution, which ensures that materials can be identified well in a scene with a low spatial resolution. A multispectral (MS) image has the opposite characteristics: it has a high spatial resolution and a low spectral resolution (Shareef et al., 2014; Bendoumi, 2012). A possible way to overcome this problem is to exploit image fusion, which merges the high spatial resolution of MS images and the high spectral resolution of HS images. Urban areas are composed of a wide range of intra-urban land covers, such as artificial and natural surface materials. Manmade features such as rooftops and road asphalt typically contain the same composition materials, thus causing considerable spectral confusion (Myint et al., 2011). According to previous studies, within-class spectral diversity can be observed among urban targets, and spectral similarities among different classes present additional difficulties in extracting detailed features (Zhou and Troy, 2008; Myint et al., 2011; Hamedianfar and Shafri, 2014b). Given these spectral heterogeneities and confusion, erroneous thematic maps created by spectral-based image classifiers are produced as a result (Hamedianfar et al., 2014c; Hamedianfar and Shafri, 2015)

Fusion-based methods require information from various sensors. Eismann and Hardie (2005) proposed a maximum a posteriori estimation method, which enhances the spatial resolution of HS images by applying the features of panchromatic or MS images. However, in this technique, the spatial enhancement is limited to several principal components of the HS image. The most initial research was based on pan sharpening, like PCA-based techniques (Licciardi et al., 2012), CN sharpening, Gram-Schmidt sharpening (Maurer, 2013), and unmixing-based fusion (Chakravortty and Subramaniam, 2014).

MS and HS sensors work in different regions of the electromagnetic spectrum; thus, band mapping another major problem arises (Khandelwal and Rajan, 2011). The spectrum range of HS images is wider than that of MS images. Fusion contains extra HS spectrum regions, which ideally should not be mapped to the MS bands. Still, some limitations exist in mapping urban areas by using HS data, such as the lack of a suitable spectral library for 
urban materials, shadow, non-roof coverage elements, and spectral similarity (Taherzadeh et al., 2011). (He et al., 2016) apply nonnegative matrix factorization (NMF) and hyperspherical color sharpening (HCS) to fuse WorldView-2 with panchromatic and multispectral images. Applying different techniques to combine multi-source RS data increase the precision of land cover classification and increase the resolution of RS data mixing fused temporal, spectral, angular, and topographic features can crop meaningfully advanced land cover classification accurateness than one RS data (Chen et al., 2017; Restaino et al., 2016).

Some materials have similar spectral signatures, thereby resulting in confusion between some roofing materials because of a lack of sufficient spectral resolution of these types. This study attempts to solve the problems of these materials by fusing HS and MS images and obtains an image with very high spectral and spatial resolutions. Hyperspectral image fusion indicates the badly-behaved of combining a HS image with another higher spatial resolution MS image to spatially localize the abundances (Mookambiga et al., 2017). Though, it is normally recognized that multisource sensor can help enhance data extraction over the usage of only one data source (Gibril et al., 2017; Hasanlou et al., 2016). The aim of this study is to extract and classify exhaustive land cover varieties in Kuala Lumpur in Malaysia, which embraces of a fusion of clearance and changed land cover which are (water, road, swimming pool and some types of roofs materials like asbestos, tarmac, metal, clay, and some vegetation types such as grass and tree species). Consequently, the goals of this study are to investigate diverse image merging techniques to recognize which type offers the full advantage of using multispectral fusion with hyperspectral data and to assess the appropriateness of relating altered classification approaches to provide a reliable LULC map.

\section{Materials and Methods}

Study Area: The study area was located in a part of Kuala Lumpur, Malaysia, and lies between longitudes $101^{\circ} 41^{\prime} 50^{\prime \prime} \mathrm{E}$ and $101^{\circ} 42^{\prime} 00^{\prime \prime} \mathrm{E}$, and latitudes $3^{\circ} 08^{\prime} 10^{\prime \prime} \mathrm{N}$ and $3^{\circ} 09^{\prime} 50^{\prime \prime} \mathrm{N}$. This area is covered by various types of impervious and pervious surfaces that show great variation in spectral and spatial characteristics, such as metal roofs, asbestos roofs, roads, grass, trees, water, and shadows.

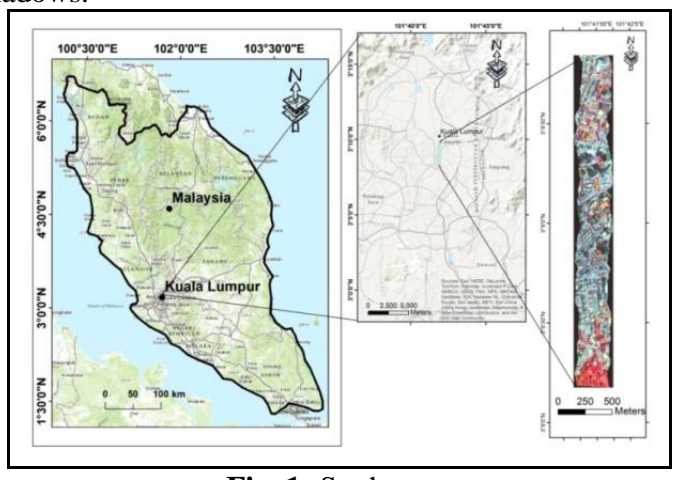

Fig. 1: Study area

Multispectral data: The WorldView-2 (WV-2) image used in this study was acquired in 2010 . WV-2 offers the first commercially available imagery with eight spectral bands (coastal, blue, green, yellow, red, red edge, NIR1, and NIR2) (Li et al., 2017). It has 11bit radiometric resolution and the highest viewing angle $\left( \pm 45^{\circ}\right)$ of any VHR imagery, which yields a $1651 \mathrm{~km}$ wide swath (Digital Globe 2010). Figure 1 shows the study area of this research.

Hyperspectral data: The AISA Eagle airborne HS sensor was deployed in 2010. The HS systems were carried by aircraft to collect the strips over Kuala Lumpur, Malaysia. The strips were recorded by using the AISA Eagle sensor in 2010. The image was collected with a $0.68 \mathrm{~m}$ spatial resolution and contained 128 spectral bands from 400 to $970 \mathrm{~nm}$ with a $\sim 5 \mathrm{~nm}$ bandwidth.

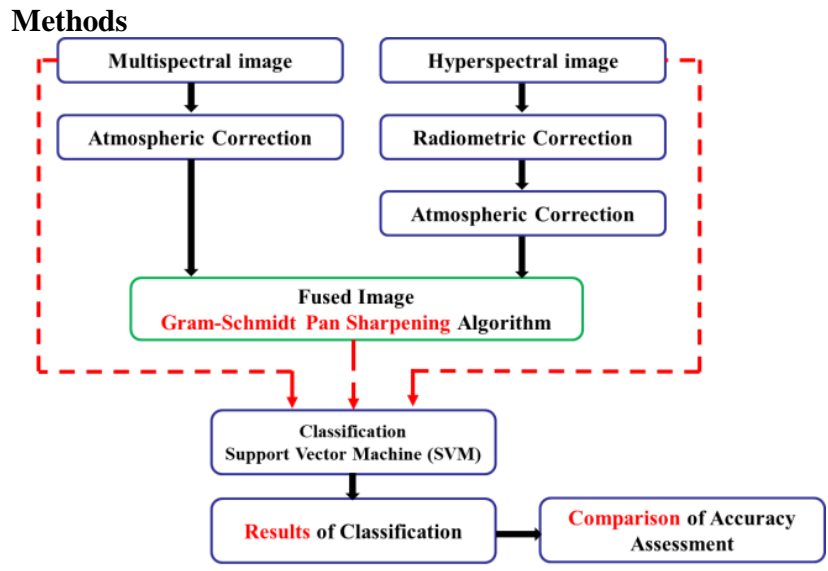

Fig. 2: Flow chart of the methodology

Categorization of urban materials: On the basis of the HS image, the test site was categorized to classify different materials in the study area. To perform this step, a field survey was conducted, and 12 classes were defined to generate a ground truth map. Training and testing pixels were determined based on random sampling and were separated to avoid bias in the accuracy assessment process.

Pre-processing: Before pan sharpening the HS and MS images, some corrections should be applied, which include atmospheric and geometric correction. In this study, due to the lack of some meteorological parameters during the flight, the Quick Atmospheric Correction (QUAC) extension was used, which is available in the ENVI 4.7 software. Applied mask on the MS image based on the shapefile was created from the HS image. Minimum noise fraction was applied for dimensionality reduction of the HS data.

Processing: The processing of this study include pan-sharpening, classification and accuracy assessment

Pan-sharpening: MS and HS images were fused with GramSchmidt pan-sharpening to sharpen MS data by using high-spatialresolution data. We applied principal component (PC) transform and the Gram-Schmidt method; the latter is more accurate than the former and is recommended for most applications. The GramSchmidt method is typically more accurate because it uses the spectral response function of a given sensor to estimate what the data look like (Maurer 2013). Table 1 shows the conversion bands from the HS image to the fused image.

Table 1: Conversion the bands from Hyperspectral image to fused image

\begin{tabular}{|c|c|c|c|}
\hline Spectral Bands of & (MS) bands & \multicolumn{2}{|c|}{ Average of (MS) bands } \\
\hline 7 - 12 bands & Costal & $\begin{array}{l}400-450 \\
n m\end{array}$ & 427 \\
\hline 13 - 25 bands & Blue & $\begin{array}{l}450-510 \\
\mathrm{~nm}\end{array}$ & 487 \\
\hline 26 - 40 bands & Green & $\begin{array}{l}510-580 \\
\mathrm{~nm}\end{array}$ & 546 \\
\hline 42 - 49 bands & Yellow & $\begin{array}{l}585-625 \\
\mathrm{~nm}\end{array}$ & 608 \\
\hline 52 - 63 bands & Red & $\begin{array}{l}630-690 \\
\mathrm{~nm}\end{array}$ & 659 \\
\hline 67 - 75 bands & Red Edge & $\begin{array}{l}705-745 \\
\mathrm{~nm}\end{array}$ & 724 \\
\hline 81 - 106 bands & Near IR1 & $\begin{array}{l}770-895 \\
\mathrm{~nm}\end{array}$ & 831 \\
\hline 101 - 128 bands & Near IR2 & $\begin{array}{l}860- \\
1040 \mathrm{~nm}\end{array}$ & 908 \\
\hline
\end{tabular}

The fused image data, which has a high spatial resolution of $0.5 \mathrm{~m}$ with 111 spectral bands, was used to identify land cover materials.

Classification: Support vector machine (SVM) represents a group of theoretically superior machine learning algorithms. The SVM employs optimization algorithms to locate the optimal boundaries between classes.

Statistically, the optimal boundaries should be generalized to unseen samples with the least errors among all possible boundaries 
separating the classes, thereby minimizing the confusion between classes.

In this paper, the SVM classification method was used in terms of using 12 classes, and the parameters of SVM are set as follows:

1. The gamma in the kernel function was set as 0.008 . This value was selected to set the gamma parameter used in the kernel function and is a floating-point value greater than 0 .

2 . The penalty parameter was set as 100 . This parameter controls the tradeoff between allowing training errors and forcing rigid margins. Increasing the value of the penalty parameter increases the cost of misclassifying points. Moreover, it causes ENVI to create a more accurate model that may not generalize well.

3 . The classification threshold was set as 0 . This field is used to set the probability that is required for the SVM classifier to classify a pixel. The pixels with rule probabilities that are less than this threshold are unclassified. The range is from 0 to 1 .

Accuracy assessment: Generally, classification accuracy refers to the extent of correspondence between the remotely sensed data and reference information (Taherzadeh et al., 2011). To assess the accuracy of land cover maps extracted from the MS, HS, and fused images, testing data provided based on the field survey were used, and the results were recorded in a confusion matrix. A nonparametric Kappa test was also used to measure the classification accuracy. In accordance with the kappa and overall accuracy assessment, we can compare the produced land cover maps.

\section{Results}

This research verified that the spatial resolution of HS can be enhanced significantly with its spectral data through fusion with MS imagery.

Spectral Comparison: The spectral profiles of the various land cover classes present in the scene area, such as vegetation, bare soil, cropland, and fallow land, and three fused products were compared among AISA HS, MS, and fused data. Figures 3, 4 and 5 show the spectral of the road in the MS, HS and fused images respectively. Furthermore, figures 6,7 and 8 show the result of SVM classification for three images MS, HS and fused images respectively.

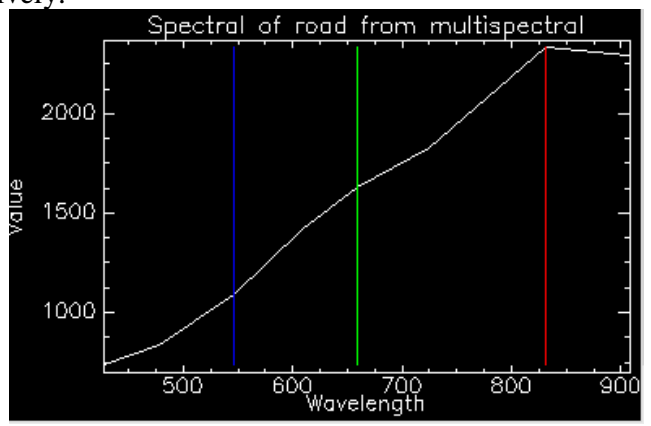

Fig. 3: Spectral of road from multispectral image

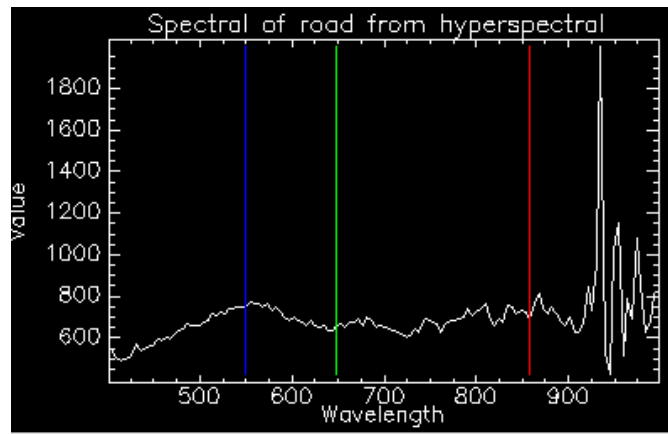

Fig. 4: Spectral of road from hyperspectral image

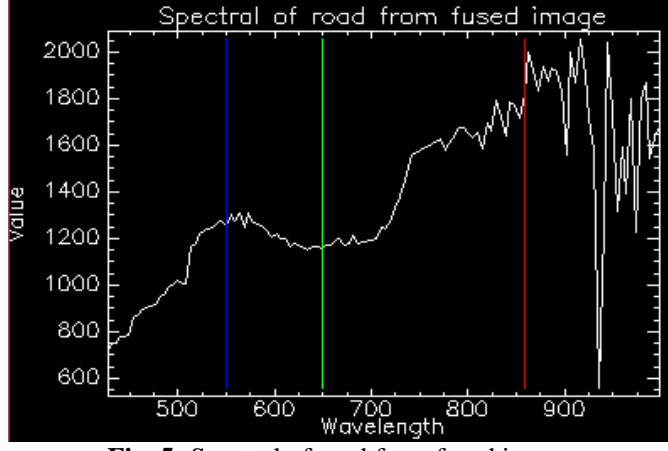

Fig. 5: Spectral of road from fused image

The outcomes illustrate that the use of the Gram-Schmidt pan sharpening algorithm for fusing HS and MS images with the SVM classification method provides suitable accuracy for detecting roof materials in urban areas. Figure 6, 7 and 8 show the result of SVM classification using MS, HS and fused images respectively.

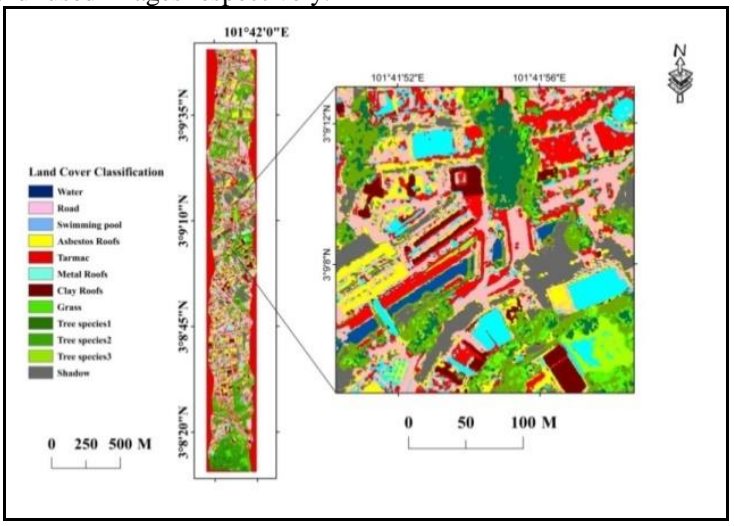

Fig. 6: Result of SVM classification using multispectral image

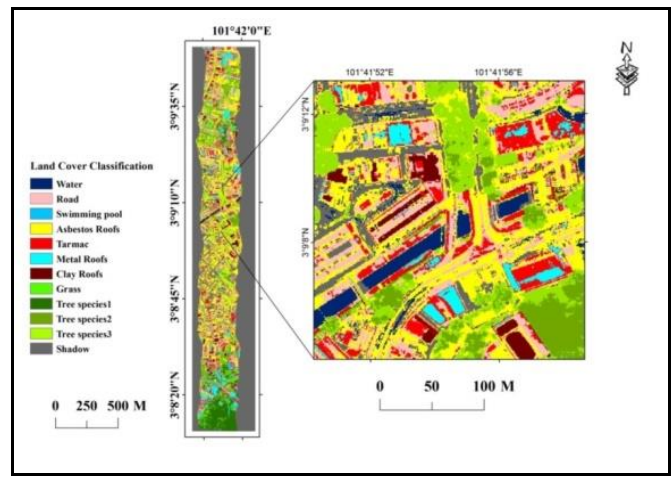

Fig. 7: Result of SVM classification using Hyperspectral image

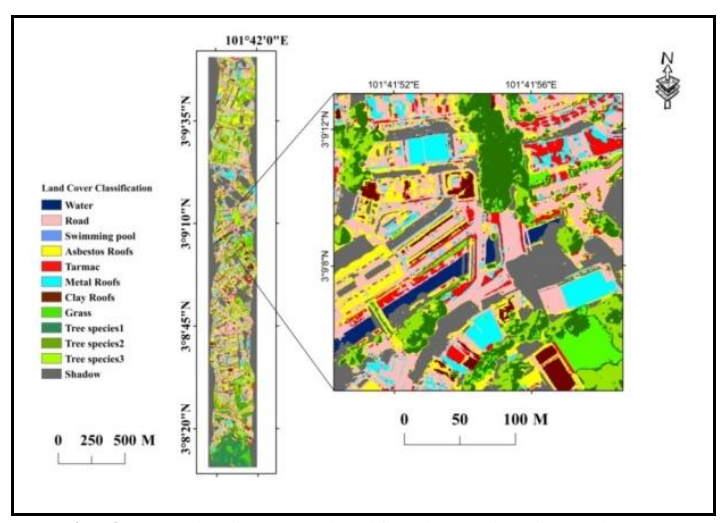

Fig. 8: Result of SVM classification using fused image

The results show that by applying Gram-Schmidt pan-sharping we can improve our classification accuracy. The maximum overall accuracy that can be achieved is $89.34 \%$ which is related to the use of the fused image. In contrast, the overall accuracy of the HS image was 72.33 when we have used SVM classification. On the 
other hand, the overall accuracy of the MS image was 82.83. Table 2 shows the overall accuracy assessment and a kappa coefficient of three maps.

Table 2: accuracy assessment of whole data

\begin{tabular}{|l|l|l|}
\hline Images & Overall accuracy & Kappa Coefficient \\
\hline Fused Image & $89.34 \%$ & 0.88 \\
\hline Hyperspectral Image & $72.33 \%$ & 0.71 \\
\hline Multispectral Image & $82.83 \%$ & 0.81 \\
\hline
\end{tabular}

In this paper as mentioned the special focus is to detect the land cover. The results show improvement in detection accuracy after applying Gram-Schmidt to fuse between HS and MS images. The details in all result of the map showed that highest user accuracy was achieved for some types of land cover materials such as water, swimming pool and some types of roofs materials like asbestos and metal roofs while lowest user accuracy was achieved for Tree species3. Table 3, 4 and 5 shows the classification accuracy assessment of MS, HS and fused maps.

Table 3: Classification accuracy of MS image

\begin{tabular}{|l|l|c|}
\hline Class & $\begin{array}{c}\text { Prod. Accuracy } \\
\text { (percent) }\end{array}$ & $\begin{array}{c}\text { User. Accuracy } \\
\text { (percent) }\end{array}$ \\
\hline Water & 97.89 & 100 \\
\hline Roads & 98.05 & 95.04 \\
\hline Swimming Pool & 69.51 & 100 \\
\hline Asbestos Roofs & 86.57 & 89.87 \\
\hline Tarmac & 100 & 85.74 \\
\hline Metal Roofs & 97.26 & 92.70 \\
\hline Clay Roofs & 97.02 & 98.11 \\
\hline Grass & 88.26 & 75.61 \\
\hline Tree species 1 & 74.74 & 73.61 \\
\hline Tree species 2 & 65.84 & 55.65 \\
\hline Tree species 3 & 34.09 & 57.72 \\
\hline Shadow & 95.06 & 100 \\
\hline
\end{tabular}

Table 4: Classification accuracy of HS image

\begin{tabular}{|l|c|c|}
\hline Class & $\begin{array}{c}\text { Prod. Accuracy } \\
\text { (percent) }\end{array}$ & $\begin{array}{c}\text { User. Accuracy } \\
\text { (percent) }\end{array}$ \\
\hline Water & 100 & 91.08 \\
\hline Roads & 88.10 & 80.83 \\
\hline Swimming Pool & 80 & 100 \\
\hline Asbestos Roofs & 87.33 & 38.21 \\
\hline Tarmac & 6.25 & 2.27 \\
\hline Metal Roofs & 88.14 & 90.64 \\
\hline Clay Roofs & 59.05 & 98.59 \\
\hline Grass & 11.69 & 14.02 \\
\hline Tree species 1 & 92.78 & 67.75 \\
\hline Tree species 2 & 71.54 & 79.03 \\
\hline Tree species 3 & 39.64 & 48.51 \\
\hline Shadow & 97.32 & 87.88 \\
\hline
\end{tabular}

Table 5: Classification accuracy of fused image

\begin{tabular}{|l|c|c|}
\hline Class & $\begin{array}{c}\text { Prod. Accuracy } \\
\text { (percent) }\end{array}$ & $\begin{array}{c}\text { User. Accuracy } \\
\text { (percent) }\end{array}$ \\
\hline Water & 100 & 93 \\
\hline Roads & 99.70 & 86.76 \\
\hline Swimming Pool & 70.03 & 100 \\
\hline Asbestos Roofs & 85.60 & 99.55 \\
\hline Tarmac & 98.13 & 94.77 \\
\hline Metal Roofs & 99.31 & 99.57 \\
\hline Clay Roofs & 96.81 & 100 \\
\hline Grass & 98.36 & 76.30 \\
\hline Tree species 1 & 94.92 & 77.11 \\
\hline Tree species 2 & 66.37 & 82.07 \\
\hline Tree species 3 & 46.53 & 58.84 \\
\hline Shadow & 99.45 & 100 \\
\hline
\end{tabular}

Discussion: Any remote sensing application that is dependent on spectral data in imagery can be expected to have been advanced significantly by our results. This research proved that the fusion of HS and MS imagery can be accomplished without the unwanted result of degradation of the HS imagery's spectral data, thereby enabling applications to be able to better utilize the spectral information in HS imagery. The fusion technique developed in this study will help meet the need of these applications for digital image processing methods for HS imagery. The fusion of HS imagery with MS imagery is achieved by implementing the GramSchmidt fusion algorithm, which is typically used. The spectral data of the HS imagery were preserved during fusion with MS imagery, thereby improving the spatial resolution. Such improvement was achieved by modifying how the Gram-Schmidt algorithm performs the component substitution. Given the success of this study in proving the feasibility of spatial resolution enhancement of HS imagery through fusion with MS imagery and its novel ability to prevent significant degradation of the HS imagery's spectral data, the method can be expected to benefit many remote sensing applications. Applications that depend on both the spectral data contained in HS imagery and the spatial resolution of MS imagery will particularly benefit from the findings of this study.

\section{Conclusion:}

Fusion aims to enhance the apparent information in images as well as to increase the reliability of the digital interpretation. Fused image data with a high spatial resolution of $0.5 \mathrm{~m}$ and 111 spectral bands were used to identify the land cover materials. We utilized a high-spectral-resolution HS imaging system that combines HS and MS sensors and presented the ground experiment for HS and MS data fusion. The use of fused data shows good potential for mapping urban areas. The application of the Gram-Schmidt pan sharpening algorithm for fusing HS and MS images with the SVM classification method provides suitable accuracy for detecting roof materials in urban areas. The details in all results in the map showed that the highest user accuracy was achieved for some land cover materials, such as water and swimming pool, and some roof materials, such as asbestos and metal. The lowest user accuracy was achieved for tree species 3 .

\section{Acknowledgment}

This study was supported by the Department of Civil Engineering, Faculty of Engineering, University Putra Malaysia. We would like to thank (Dr. Alireza Hamedianfar) for his help and anonymous reviewers and the editor for providing valuable suggestions

\section{References}

[1] Ajaj, Q.M., Pradhan, B., Noori, A.M. and Jebur, M.N., 2017. Spatial Monitoring of Desertification Extent in Western Iraq using Landsat Images and GIS. Land Degradation \& Development, 28(8):2418-2431.

[2] Bendoumi, M.A., He, M., Mei, S. and Zhang, Y., 2012, December. Unmixing approach for hyperspectral data resolution enhancement using high resolution multispectral image. In Control Automation Robotics \& Vision (ICARCV), 2012 12th International Conference on:1369-1373. IEEE.

[3] Chen, B., Huang, B. and Xu, B., 2017. Multi-source remotely sensed data fusion for improving land cover classification. ISPRS Journal of Photogrammetry and Remote Sensing, 124, :27-39.

[4] Chakravortty, S. and Subramaniam, P., 2014. Fusion of Hyperspectral and Multispectral Image Data for Enhancement of Spectral and Spatial Resolution. The International Archives of Photogrammetry, Remote Sensing and Spatial Information Sciences, 40(8), p.1099.

[5] DigitalGlobe. 2010. Digital Globe Core Imagery Products Guide. Accessed October

2013.http://www.digitalglobe.com/downloads/DigitalGlobe_Core_I magery_Products_Guide.pdf.

[6] Eismann, M.T. and Hardie, R.C., 2005. Hyperspe -ctral resolution enhancement using high-resol -ution multispectral imagery with arbitrary response functions. IEEE Transactions on Geoscience and Remote Sensing, 43(3):455-465.

[7] Gibril, M.B.A., Bakar, S.A., Yao, K., Idrees, M.O. and Pradhan, B., 2017. Fusion of RADARSAT-2 and multispectral optical remote sensing data for LULC extraction in a tropical agricultural area. Geocarto international, 32(7):735-748. 
[8] Hamedianfar, A., Shafri, H.Z.M., Mansor, S. and Ahmad, N., 2014a. Combining data mining algorithm and object-based image analysis for detailed urban mapping of hyperspectral images. Journal of Applied Remote Sensing, 8(1), p.085091.

[9] Hamedianfar, A. and Shafri, H.Z., 2014b. Development of fuzzy rule-based parameters for urban object-oriented classification using very high resolution imagery. Geocarto International, 29(3):268292.

[10] Hamedianfar, A., Shafri, H.Z.M., Mansor, S. and Ahmad, N., 2014c Improving detailed rule-based feature extraction of urban areas from WorldView-2 image and lidar data. Internation

[11] Hamedianfar, A. and Shafri, H.Z.M., 2015. Detailed intra-urban mapping through transferable OBIA rule sets using WorldView-2 very-high-resolution satellite images. International Journal of Remote Sensing, 36(13):3380-3396

[12] He, G., Xing, S., Xia, Z., Dong, D. and Wei, Y., 2016, December Study on WorldView-2 Image Fusion Method Based on NMF and HCS Transform. In Internet of Things (iThings) and IEEE Green Computing and Communications (GreenCom) and IEEE Cyber, Physical and Social Computing (CPSCom) and IEEE Smart Data (SmartData), 2016 IEEE International Conference on :548-553. IEEE.

[13] Hasanlou, M. and Saradjian, M.R., 2016. Quality assessment of pan-sharpening methods in high-resolution satellite images using radiometric and geometric index. Arabian Journal of Geosciences, 9(1), p.45.

[14] Khandelwal, A. and Rajan, K.S., 2011, July. Hyperspectral image enhancement based on sensor simulation and vector decomposition. In Information Fusion (FUSION), 2011 Proceedings of the 14th International Conference on (pp. 1-6). IEEE.

[15] Licciardi, G.A., Khan, M.M., Chanussot, J., Montanvert, A., Condat, L. and Jutten, C., 2012. Fusion of hyperspectral and panchromatic images using multiresolution analysis and nonlinear PCA band reduction. EURASIP Journal on Advances in Signal processing, 2012(1), p.207.

[16] Li, H., Jing, L. and Tang, Y., 2017. Assessment of pansharpening methods applied to worldview-2 imagery fusion. Sensors, 17(1) p.89.

[17] Maurer, T., 2013. How to pan-sharpen images using the GramSchmidt pan-sharpen method-a recipe. International archives of the photogrammetry, remote sensing and spatial information sciences, 1 , p.W1.

[18] Mookambiga, A. and Gomathi, V., 2016. Compreh -ensive review on fusion techniques for spatial information enhancement in hyperspectral imagery. Multidimensional Systems and Signal Processing, 27(4):863-889.

[19] Myint, S.W., Gober, P., Brazel, A., Grossman-Clarke, S. and Weng, Q., 2011. Per-pixel vs. object-based classification of urban land cover extraction using high spatial resolution imag -ery. Remote sensing of environment , 115(5):1145-1161.

[20] Shareef, M.A., Toumi, A. and Khenchaf, A., 2014. Estimation of water quality parameters using the regression model with fuzzy kmeans clustering. international journal of advanced computer science and applications, 5, p.xx.

[21] Taherzadeh, E. and Shafri, H.Z., 2011, April. Using hyperspectral remote sensing data in urban mapping over Kuala Lumpur. In Urban Remote Sensing Event (JURSE), 2011 Joint : 405-408. IEEE.

[22] Woycheese, J.P., Pagni, P.J. and Liepmann, D., 1998. Brand lofting above large-scale fires. Building and Fire Research Laboratory, National Institute of Standards and Technology.

[23] Restaino, R., Vivone, G., Dalla Mura, M. and Chanussot, J., 2016 Fusion of multispectral and panchromatic images based on morphological operators. IEEE Transactions on Image Processing, 25(6):2882-2895.

[24] Zhou, W. and Troy, A., 2008. An object-oriented approach for analysing and characterizing urban landscape at the parcel level. International Journal of Remote Sensing, 29(11):3119-3135. 